\title{
Hydrometeor classification from dual-polarized weather radar: extending fuzzy logic from S-band to $\mathrm{C}$-band data
}

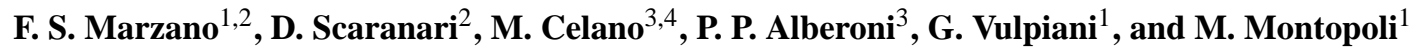 \\ ${ }^{1}$ CETEMPS, Università dell'Aquila, L'Aquila, Italy \\ ${ }^{2}$ DIE, Università degli Studi di Roma "La Sapienza”, Rome, Italy \\ ${ }^{3}$ ARPA-SIM, Bologna, Italy \\ ${ }^{4}$ DF, Università di Ferrara, Ferrara, Italy
}

Received: 11 November 2005 - Revised: 16 January 2006 - Accepted: 30 January 2006 - Published: 14 February 2006

\begin{abstract}
A model-based fuzzy classification method for Cband polarimetric radar data, named Fuzzy Radar Algorithm for Hydrometeor Classification at C-band (FRAHCC), is presented. Membership functions are designed for best fitting simulation data at C-band, and they are derived for ten different hydrometeor classes by means of a scattering model, based on T-Matrix numerical method. The fuzzy logic classification technique uses a reduced set of polarimetric observables, i.e. copolar reflectivity and differential reflectivity, and it is finally applied to data coming from radar sites located in Gattatico and S. Pietro Capofiume in North Italy. The final purpose is to show qualitative accuracy improvements with respect to the use of a set of ten bidimensional MBFs, previously adopted and well suited to S-band data but not to C-band data.
\end{abstract}

\section{Introduction}

New generation of dual-polarized weather radar systems may offer the opportunity to detect and identify different classes of hydrometeors present in stratiform and convective storms (Bringi and Chandrasekar, 2001). This important feature depends on the fact that polarimetric radar measurements are highly sensitive to physical properties of hydrometeors like composition, size, shape and orientation (Vulpiani et al., 2005). Hydrometeor classification may facilitate to study rain-cloud microphysics, to detect hailstorms and to choose the correct algorithm for precipitation rate retrieval. Last but not least, hydrometeor identification may also be useful for flight assistance and weather nowcasting.

Polarimetric signatures depend also on the frequency used by the radar system. The consequence is that S-band signals differ substantially from C-band signals, especially as

Correspondence to: F. S. Marzano

(marzano@die.uniroma1.it) far as differential reflectivity and specific differential phase are concerned. As a matter of fact, most scientific literature about hydrometeor classification describes classification techniques designed for S-band radar data (e.g., Vivekanandan et al., 1999; Straka et al., 2000; Zrnić et al., 2001; Lim et al., 2005). Only recently some works related to C-band measurements have been presented (Alberoni et al., 2002; Keenan, 2003; Baldini et al., 2004; Galletti et al., 2005). The interest of assessing C-band hydrometeor classification emerges from the consideration that almost all European weather radars operate at C-band. C-band radar systems have a reduced antenna size and, usually, an overall lower cost with respect to that of an S-band system with similar characteristics (Baldini et al., 2004).

In this work, a model-based fuzzy-logic classification method for C-band polarimetric radar data is presented. Membership functions (MBFs) are designed for best fitting simulation data at C-band and they are derived for ten different hydrometeor classes by means of a radar scattering model, based on T-Matrix numerical method. The fuzzy logic classification technique, named Fuzzy Radar Algorithm for Hydrometeor Classification at C band (FRAHCC), uses here a reduced set of polarimetric observables, i.e. $Z_{h h}$ and $Z_{d r}$, and it is finally applied to data coming from radar sites located in Gattatico and S. Pietro Capofiume in Northern Italy. The main purpose is to show qualitative accuracy improvements with respect to the use of a set of ten bidimensional MBFs, previously adopted and well suited for S-band dual-polarized data, but not for C-band dual-polarized data.

\section{Hydrometeor radar scattering model}

Since retrieval of in situ hydrometeor data regarding composition of storms is not a simple task, a hydrometeor ensemble scattering model, based on the T-Matrix method (Barber and Yeh, 1975), can be used in order to obtain hydrometeor 


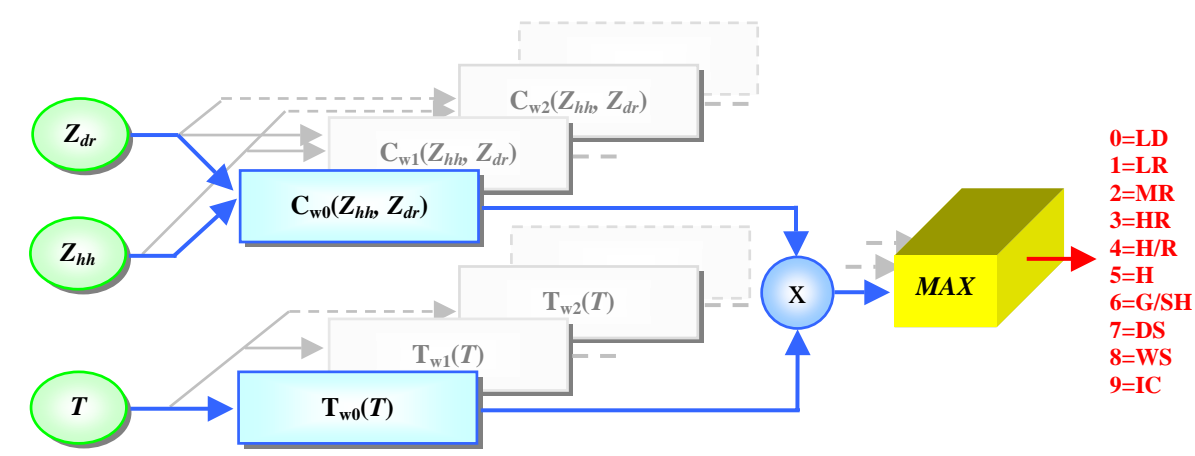

Fig. 1. Schematic flow diagram of the Fuzzy Radar Algorithm for Hydrometeor Classification at C-band (FRAHCC). The fuzzy logic scheme for hydrometeor classification is illustrated with a reduced set of polarimetric radar observables $\left(Z_{h h}, Z_{d r}\right)$ and temperature $(T)$ as inputs. The hydrometeor classes are defined in the text.

polarimetric signatures and their relation with hydrometeor properties (Vulpiani et al., 2005). T-Matrix method is also known as EBCM, Extended Boundary Condition Method, and it is the theoretical solution of the scattering problem for non spherical particles by means of the electromagnetic equivalence theorem (Mishchenko and Travis, 1998).

Horizontally-polarized reflectivity (factor) $Z_{h h}$ is defined as:

$Z_{h h}=\frac{\lambda^{4}}{\pi^{5}|K|^{2}} \int_{0}^{\infty} \sigma_{b, h h}(D) N(D) d D=\frac{\lambda^{4}}{\pi^{5}|K|^{2}}<\sigma_{b, h h}>$

being $|K|^{2}$ the dielectric factor (its value for water is about 0.93 from S-band to X-band), whereas $D$ is the diameter and $N(D)$ represents the particle size distribution (PSD) for a specific hydrometeor. In (1) $\sigma_{b}$ is the backscattering cross section, whereas the angle brackets stand for ensemble averaging over $N(D)$. The differential reflectivity $Z_{d r}$ and specific differential phase $K_{d p}$ are given by:

$Z_{d r}=10 \log _{10}\left(\frac{Z_{h h}}{Z_{v v}}\right)=10 \log _{10}\left(\frac{\left\langle\sigma_{b, h h}\right\rangle}{\left\langle\sigma_{b, v v}\right\rangle}\right)$

$K_{d p}=10^{-3} \frac{180}{\pi} \lambda \operatorname{Re}\left\{\int_{0}^{\infty} N(D)\left[f_{h h}(r, D)-f_{v v}(r, D)\right] d D\right\}$

where $Z_{d r}$ is here measured in $\mathrm{dB}, K_{d p}$ in $\mathrm{deg} / \mathrm{km}, f_{h h, v v}$ is the forward scattering amplitude at horizontal and vertical polarization, whereas $D$ and $\lambda$ are here expressed in $\mathrm{mm}$.

A correct physical and dielectric modelling of hydrometeors is essential to obtain simulations of polarimetric radar measurements. Useful information about several hydrometeor types can be found in Straka et al. (2000). Hydrometeor models together with environmental temperature information are included in the model simulation. Temperature is a fundamental variable in hydrometeor classification schemes: some classes can physically exist at certain temperatures, others can not. Possible temperature ranges for ten different hydrometeor classes are derived from Zrnić et al. (2001).

Scattering simulations have been carried out with temperature randomly varying in intervals specified inside the code in order to have statistically significant simulated polarimetric signatures. Temperature for every radar bin is evaluated by means of a vertical radio-sounding profile realized in proximity of the storm. In absence of this kind of measure, a standard temperature gradient can be assumed in an approximate way.

\section{Fuzzy classification method for C-band data}

Radar measurements are affected by thermal noise and power path-attenuation problems. At C-band path attenuation is relevant and cannot be neglected. Furthermore, data provided by radar measurements or T-Matrix numerical simulations tend to overlap significantly on the plane of $Z_{h h}$ and $Z_{d r}$ observations. All these aspects make fuzzy logic one of the best solutions for the hydrometeor classification problem (Bringi and Chandrasekar, 2001; Zrnić et al., 2001). Use of simulated data also allows robustness tests to be performed, adding noise with increasing standard deviations to data; in this respect, fuzzy logic performs well and its behavior tends to be robust to noise.

Polarimetric radars have dual-polarization capabilities and can retrieve reflectivity and differential reflectivity signals: these observables together with temperature information are the three inputs to the fuzzy classification scheme, as illustrated by Fig. 1 which describes the basics of FRAHCC approach.

A fuzzy logic system provides a non linear mapping of input data vectors into scalar outputs (Mendel, 1995). The input data vector is made up of reflectivity $Z_{h h}$, differential reflectivity $Z_{d r}$, and environmental temperature $T$. The fuzzifier consists of ten bidimensional MBFs for $Z_{h h}$ and $Z_{d r}$ and ten monodimensional MBFs for temperature. A priori established hydrometeor classes are, in fact, ten: LD (large drops), LR (light rain), MR (medium rain), HR (heavy rain), 
Table 1. Contingency table for evaluating the errors due to a fuzzy classification, designed at S-band and applied to C-band synthetic data. Only $Z_{h h}, Z_{d r}$ and $T$ are inputs to the classifier. Standard errors are equal to $1 \mathrm{dBZ}$ and $0.3 \mathrm{~dB}$ for $Z_{h h}$ and $Z_{d r}$, respectively (see text for details).

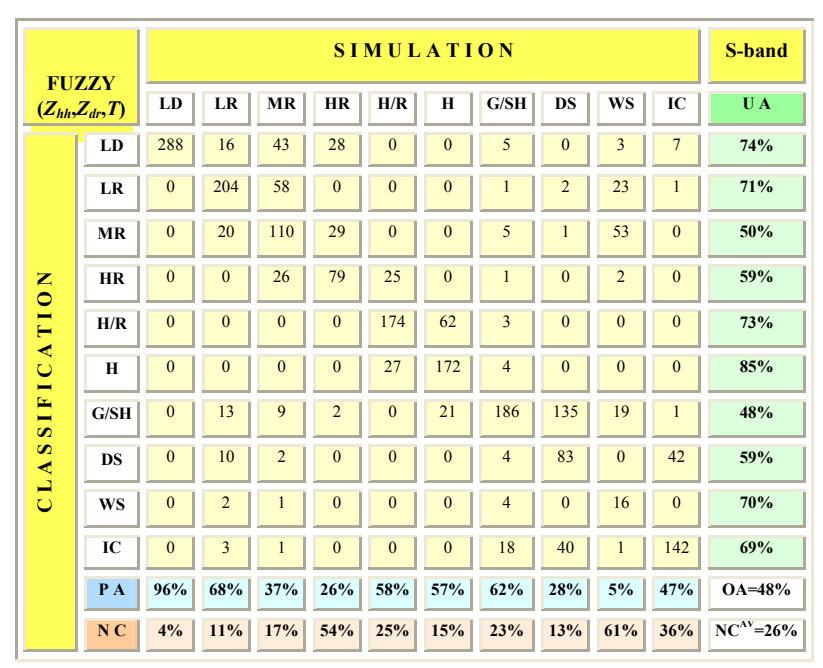

H/R (hail/rain mixture), H (hail), G/SH (graupel/small hail), DS (dry snow), WS (wet snow), IC (ice crystals). The output of the MBFs are called membership degrees and can assume values ranging from 0 (no membership) to 1 (maximum membership). Definition of MBFs is a fundamental task that affects classification accuracy.

The starting point was a set of MBFs originally described by Straka et al. (2000), and it is usually referred as the fuzzy logic scheme developed at the NSSL polarimetric radars (Zrnić et al., 2001). The scheme was designed for S-band data so that it cannot be applied in straightforward way to $\mathrm{C}$-band radar data. The latter are usually more sensitive to shapes and present bigger values of differential reflectivity (Celano et al., 2005) and specific differential phase, with respect to S-band measurements. Resonance effects due to Mie scattering cannot be neglected at C-band and MBFs must be modified.

MBFs adopted in this work have trapezoidal forms, and analytical expressions have been derived by means of accurate observations of T-Matrix simulations at $5.6 \mathrm{GHz}$, typical frequency of C-band radar systems. Most relevant differences between the two bands behaviors were found for LD, MR, HR and H/R classes. Linear fuzzy thicknesses can be different for each hydrometeor class. Temperature MBFs are trapezoidal functions, directly derived from Zrnić et al. (2001). Analytical expressions for bidimensional MBFs related to C-band observables $Z_{h h}$ and $Z_{d r}$ and for monodimensional MBFs related to environmental temperature $T$ are similar to those of Zrnić et al. (2001).

The inference rule (IR) is based on the product of membership degrees derived from MBFs, as it follows:

$$
I R_{i}=C_{w i}\left(Z_{h h}, Z_{d r}\right) \cdot T_{w i}(T), \quad i=0,1, \ldots, 9
$$

Table 2. Contingency table for evaluating the errors due to a FRAHCC fuzzy classification, designed at C-band and applied to C-band synthetic data. Only $Z_{H}, Z_{d r}$ and $T$ are inputs to the classifier. Standard errors are equal to $1 \mathrm{dBZ}$ and $0.3 \mathrm{~dB}$ for $Z_{h h}$ and $Z_{d r}$, respectively (see text for details).

\begin{tabular}{|c|c|c|c|c|c|c|c|c|c|c|c|c|}
\hline \multirow{2}{*}{\multicolumn{2}{|c|}{$\begin{array}{c}\text { FUZZY } \\
\left(Z_{h h}, Z_{d r}, T\right)\end{array}$}} & \multicolumn{10}{|c|}{ S I M UL A T I O N } & \multirow{3}{*}{\begin{tabular}{|c|} 
C-band \\
U A \\
$92 \%$ \\
\end{tabular}} \\
\hline & & \multirow{2}{*}{\begin{tabular}{|l|} 
LD \\
298
\end{tabular}} & \multirow{2}{*}{$\begin{array}{c}\text { LR } \\
9\end{array}$} & \multirow{2}{*}{\begin{tabular}{|c|} 
MR \\
5
\end{tabular}} & \multirow{2}{*}{\begin{tabular}{|c|} 
HR \\
4
\end{tabular}} & \multirow{2}{*}{\begin{tabular}{c|}
$\mathbf{H} / \mathbf{R}$ \\
0
\end{tabular}} & \multirow{2}{*}{$\begin{array}{l}\mathbf{H} \\
0\end{array}$} & \multirow{2}{*}{\begin{tabular}{|c|} 
G/SH \\
2
\end{tabular}} & \multirow{2}{*}{\begin{tabular}{|c|} 
DS \\
0
\end{tabular}} & \multirow{2}{*}{\begin{tabular}{|c|} 
WS \\
0 \\
\end{tabular}} & \multirow{2}{*}{\begin{tabular}{|c|} 
IC \\
6
\end{tabular}} & \\
\hline \multirow{12}{*}{ 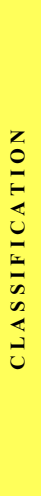 } & LD & & & & & & & & & & & \\
\hline & LR & 0 & 209 & 65 & 0 & 0 & 0 & 1 & 2 & 24 & 2 & $69 \%$ \\
\hline & MR & 0 & 20 & 134 & 35 & 0 & 0 & 5 & 1 & 55 & 0 & $54 \%$ \\
\hline & HR & 0 & 0 & 35 & 235 & 6 & 0 & 2 & 0 & 2 & 0 & $84 \%$ \\
\hline & $\mathbf{H} / \mathbf{R}$ & 0 & 0 & 0 & 3 & 246 & 52 & 4 & 0 & 0 & 0 & $81 \%$ \\
\hline & H & 0 & 0 & 0 & 0 & 29 & 194 & 5 & 0 & 0 & 0 & $85 \%$ \\
\hline & G/SH & 0 & 13 & 11 & 3 & 0 & 21 & 185 & 132 & 19 & 1 & $48 \%$ \\
\hline & DS & 0 & 11 & 2 & 0 & 0 & 0 & 5 & 95 & 0 & 40 & $62 \%$ \\
\hline & ws & 0 & 2 & 1 & 0 & 0 & 0 & 4 & 0 & 16 & 0 & $70 \%$ \\
\hline & IC & 0 & 3 & 1 & 0 & 0 & 0 & 20 & 38 & 1 & 241 & $79 \%$ \\
\hline & $\mathbf{P A}$ & $99 \%$ & $70 \%$ & $45 \%$ & $78 \%$ & $82 \%$ & $65 \%$ & $62 \%$ & $32 \%$ & $5 \%$ & $80 \%$ & $\mathrm{OA}=62 \%$ \\
\hline & NC & $1 \%$ & $11 \%$ & $15 \%$ & $7 \%$ & $6 \%$ & $11 \%$ & $22 \%$ & $11 \%$ & $61 \%$ & $3 \%$ & $\mathrm{NC}^{\mathrm{AV}}=15 \%$ \\
\hline
\end{tabular}

where the combined $C_{w i}$ and temperature $T_{w i}$ weighting MBFs properly defined. The choice of product instead of linear combination has the purpose to limit classification errors as far as possible: if, for a given class, one measurement is significantly out of range, the low value of corresponding MBF will definitely suppress the class (Baldini et al., 2004). Temperature MBFs are of primary importance because they take in account peculiar physical characteristics of hydrometeors. Maximum rule value finally gives the hydrometeor class index to which the radar bin is assigned. If two or more rules present the same maximum value, NC (not classified) label is assigned to the radar bin.

Contingency tables, also known as confusion matrices, are used to evaluate classification accuracy on either real or synthetic data (Lillesand and Kiefer, 1994). In our case "truth" data consist of 300 simulations of $Z_{h h}$ and $Z_{d r}$ for each hydrometeor class, with environmental temperature uniformly distributed inside specific intervals. Radar measurements have been simulated by adding gaussian noise to data, $1 \mathrm{dBZ}$ standard deviation for $Z_{h h}$ and $0.2 \mathrm{~dB}$ for $Z_{d r}$. Classification results for $\mathrm{C}$-band simulated radar data using previous S-band MBFs are reported in Table 1, while the contingency table for C-band data, when new MBFs C-band adapted are used within the FRAHCC scheme, is reported in Table 2. Accuracy improvements, measured in terms of overall accuracy $(\mathrm{OA})$ and average not classified bins percentage $\left(\mathrm{NC}^{A V}\right)$, are absolutely evident.

Better results could be obtained, of course, using $K_{d p}$ extrapolations from phase-shift $\Phi_{d p}$ measurements, if available (Zrnić et al., 2001). 

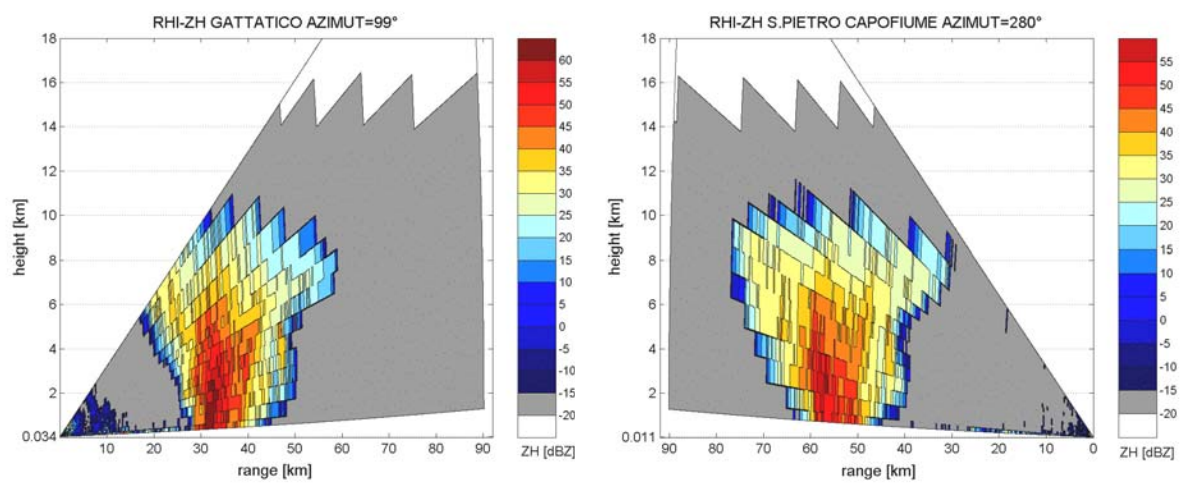

Fig. 2. Vertical section (Range Height Indicator: RHI) of copolar reflectivity $Z_{h h}$ with respect to the line of sight between the two C-band radar systems in Italy on 19 May 2003 at 16:31. Gattatico (GAT) radar is on the left side and S. Pietro Capofiume (SPC) radar on the right side and the distance between the two is about $90 \mathrm{~km}$.

\section{C-band dual-polarized radar data}

Available radar data refer to a convective episode occurred in the region between two dual-polarized C-band systems, both located in the Po valley and about $90 \mathrm{~km}$ apart: the S. Pietro Capofiume (SPC) and the Gattatico (GAT) radars, both managed by ARPA-SIM Emilia-Romagna (Alberoni et al., 2001).

\subsection{Radar systems}

These dual-polarization GPM-500C radar are placed on a tower with a Cassegrain parabolic antenna (without radome cover), providing a half-power beam-width of $1.0^{\circ}$ and a directivity of about $45-\mathrm{dB}$. The klystron peak-power is $250 \mathrm{~kW}$ at $5.6 \mathrm{GHz}$ with an alternating horizontal-vertical polarization transmission and dual pulse repetition frequency (PRF) system for unfolding capability. Pulse widths are of $0.5 \mathrm{~ms}$ (i.e., short pulse with a resampled bin resolution of $250 \mathrm{~m}$ ) and $1.5 \mathrm{~ms}$ (i.e., long pulse with a resampled bin resolution of $1500 \mathrm{~m})$. The receiver sensitivity is equal to $-113 \mathrm{dBm}$. The typically used maximum range is $250 \mathrm{~km}$ (with long pulse) and $125 \mathrm{~km}$ (with short pulse) for the intensity and velocity mode, respectively. A self-contained software is used to remotely operate and archive radar data of measured $Z_{h h}$ and $Z_{d r}$, being $K_{d p}$ not available as output.

Radar data are acquired with a prescribed scanning strategy during operational activity, consisting of 15 elevations with an angular spacing of $1^{\circ}$. Radial spatial resolution is set to $250 \mathrm{~m}$ for short ranges (i.e., $125 \mathrm{~km}$ ) and to $1000 \mathrm{~km}$ for long range (i.e., $250 \mathrm{~km}$ ) scans, the latter being carried out only twice per hour. Time sampling of radar volume data is such that there are 4 acquisitions per hour (i.e., every $15 \mathrm{~min}$ ), the dual-polarized one being performed only twice per hour. Procedures to correct for gas absorption, to remove groundclutter echoes and to identify anomalous propagation conditions are routinely applied (Alberoni et al., 2001). Side-lobe effects at short ranges (less than $20 \mathrm{~km}$ ) for low elevations are avoided by choosing higher elevations not affected by this disturbance.

\subsection{Case study}

During the night between the 19 and 20 May 2003 a cold front, arriving from North-West and moving across the Alps, caused a deep convective event in the flat lands of North-East Italy.

The reference line connecting the two radar systems was interested by the hailstorm and the storm core, characterized by high values of reflectivity (50-60 dBZ), was at about 55$60 \mathrm{~km}$ from SPC and 30-35 km from GAT. Reflectivity vertical sections, with respect to the connection line, are reported in Fig. 2. Sections refer to data acquired on 19 May 2003 at 16:31. The distance between the two radars is about $90 \mathrm{~km}$ and, close to the SPC site, a radiosounding station is operated. The latter is used for inferring the thermodynamic structure of the observed atmosphere.

Figure 2 displays the range-height-indicator (RHI) of the reflectivity $Z_{h h}$ for GAT and SPC radars on the reference line, namely the line connecting the position of the two radars. The core of the storm, characterized by high values of $Z_{h h}(50-60 \mathrm{dBZ})$, is located at about 55-60 km from SPC and about $30-35 \mathrm{~km}$ from GAT radar. From $Z_{d r}$ maps (not shown for brevity) it is possible to note the different attenuations zones in the two regions behind the convective core, depending on the different viewing of the radars. The attenuation signature is clearly highlighted by analyzing the first elevation rays of $Z_{h h}$ and $Z_{d r}$ from each radar along the reference line connecting the two radars. No attempt to correct for path attenuation has been carried out in this work, even though the bistatic radar view may be exploited to this aim.

\section{Hydrometeor classification at C-band}

Reflectivity and differential reflectivity data available from the two radars GAT and SPC have been classified with the previously described FRAHCC technique. Temperature vertical average profile has been retrieved in an approximate way from a vertical radio-sounding made at SPC meteorological station. 

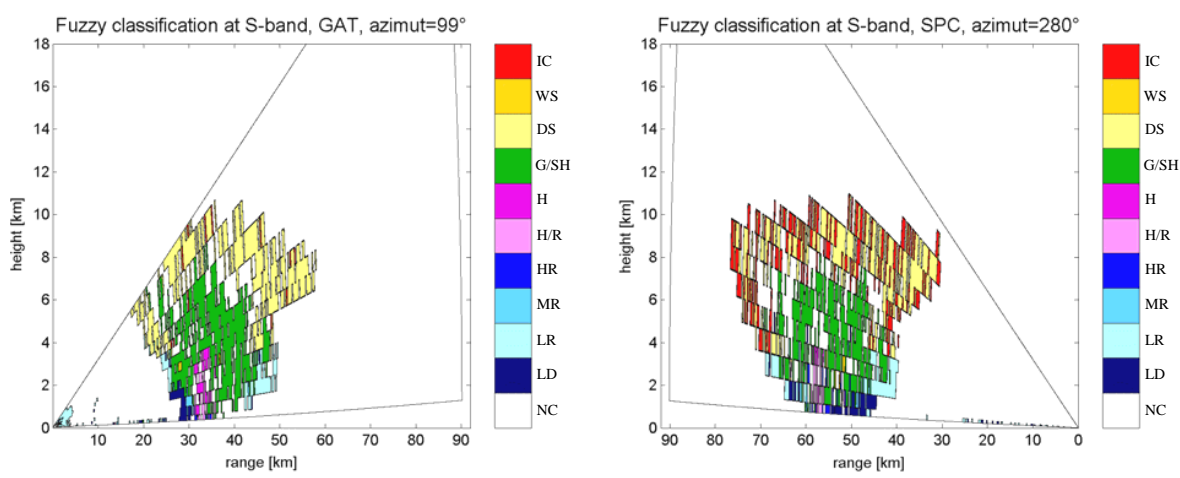

Fig. 3. Fuzzy-logic classifications using S-band membership functions (MBFs) applied to C-band radar data, displayed in Fig. 2 along the line of sight between the two radar systems.
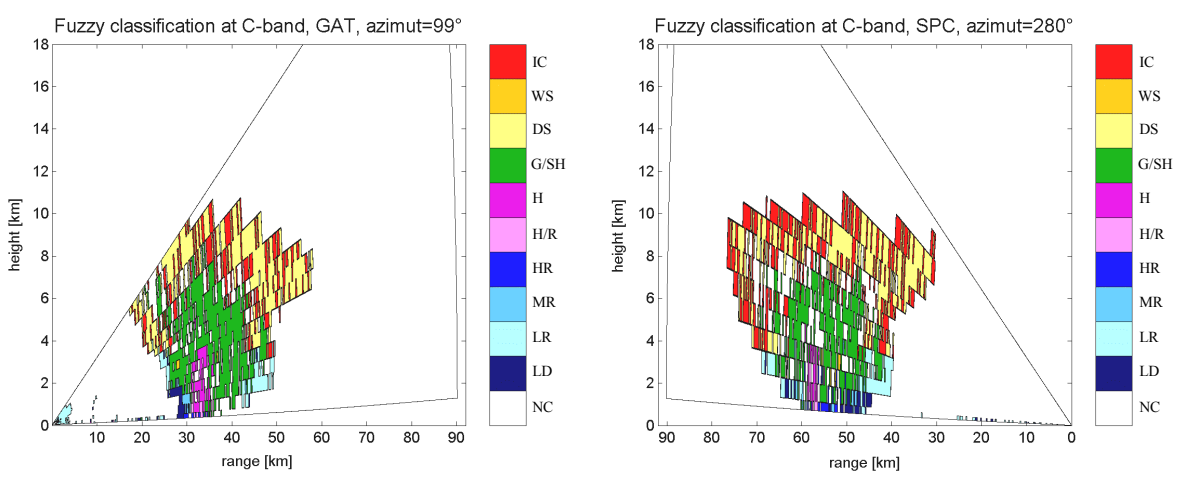

Fig. 4. Fuzzy-logic classification, using FRAHCC algorithm with proper C-band MBFs and applied to C-band radar data, displayed in Fig. 2 along the line of sight between the two radar systems.

Since truth data regarding the storm on the connection line are not available, only qualitative tests can be carried out. Classification results obtained by conventional S-band designed MBFs are shown in Fig. 3, while results obtained by new C-band adapted MBFs (i.e., FRAHCC) are reported in Fig. 4.

Accuracy improvements can be recognized by direct observations of the RHI diagrams, first with S-band MBFs and then with C-band MBFs: number of not classified (NC, white) radar bins considerably decreases, especially for GAT radar measurements, and ice crystals detection (IC, red) is now performed in a more realistic way. Classification results are also physically self-consistent: the hail core $(\mathrm{H}$ and $\mathrm{H} / \mathrm{R}$, pink and violet) is correctly detected at the centre of the convective storm, graupel $(\mathrm{G} / \mathrm{SH}$, green) is reasonably identified all around the hail core, rain is present only at low altitude (different shades of blue) while snow and ice crystals (DS, WS, IC, from yellow to red) are properly found at the top of the storm.

As noted, better results could be obtained with $K_{d p}$ measurements and with attenuation correction techniques applied to the measured reflectivity signals. A composite maximumreflectivity approach (i.e., using co-locating reflectivity data and choosing the maximum value) could be used to correct the whole vertical section radar data for path attenuation effects (Celano et al., 2005).

\section{Conclusions}

Backscattering radar simulations confirm the presence of noteworthy behavioral differences between S-band and Cband data. At C-band Mie-scattering resonance effects cannot be neglected and the consequence is a major sensitivity to shapes and orientations, with respect to S-band signals. Using S-band MBFs to classify C-band simulated data naturally induces a poor classification accuracy. Appropriate C-band modified MBFs, constituting the basics of the FRAHCC approach, must be defined and, when applied to available data, they seem to perform reasonably well. Making changes is not straightforward: it requires a solid experience and accurate observations of hydrometeor polarimetric signatures on $Z_{h h}-Z_{d r}$ plane, derived from T-Matrix simulations, must be performed in order to modify the internal contour lines of the trapezoidal functions. New C-band MBFs have been tested on simulated radar measurements, obtained adding Gaussian noise to simulated data: overall accuracy considerably increases and average not classified bins percentage decreases as well.

Finally, C-band fuzzy-logic FRAHCC classification has been applied to data coming from Gattatico and S. Pietro Capofiume radar sites, located in North Italy. The vertical section of a convective hailstorm on the line of sight between the two radar systems is classified with the old fuzzy system 
and with the new modified system: accuracy improvements are directly visible, the hail core is correctly detected and the classification results are physically reasonable. Future work shall be devoted to the improvement of classification accuracy with the use of $K_{d p}$ measurements, still not available on GPM-500C systems, and to the devise of a path-attenuation correction algorithm applied to the entire vertical section of the precipitating cloud. Preliminary results given by the hybrid fuzzy logic technique with $K_{d p}$ simulated measurements are quite promising and the bistatic radar configuration can be surely exploited to this aim.

Acknowledgements. This work has been funded by Civil Protection Directorate of Region Abruzzo, Italy, and by RISK-AWARE project within INTERREG-IIIB CADSES framework.

Edited by: V. Kotroni and K. Lagouvardos

Reviewed by: anonymous referee

\section{References}

Alberoni, P. P, Andersson, T., Mezzasalma, P., Michelson, D. B, and Nanni, S.: Use of the vertical reflectivity profile for identification of anomalous propagation, Meteorol. Appl., 8, 257-266, 2001.

Alberoni, P. P., Zrnić, D. S., Ryzhkov, A. V., and Guerrieri, L.: Use of a fuzzy logic classification scheme with a C-band polarimetric radar: first results, Proceedings of ERAD, pp. 324-327, 2002.

Baldini, L., Gorgucci, E., and Chandrasekar, V.: Hydrometeor classification methodology for C-band polarimetric radars, Proceedings of ERAD, pp. 62-66, 2004.

Barber, P. W. and Yeh, C.: Scattering of electromagnetic waves by arbitrarily shaped dielctric bodies, Appl. Opt., 14, 12, 28642872, 1975.

Bringi, V. N. and Chandrasekar, V.: Polarimetric Doppler weather radar, Cambridge University Press, 2001.
Celano, M., Alberoni, P. P., Levizzani, V., and Holt, A. R.: Analysis of severe convective event from two operational dual polarisation doppler radars, EGU General Assembly, 24-29 April, no. 671, 2005.

Galletti, M., Alberoni, P. P., Levizzani, V., and Celano, M.: Assessment and tuning of the behaviour of a microphysical characterisation scheme, Adv. Geosci., 2, 145-150, 2005.

Keenan, T. D.: Hydrometeor classification with a C-band polarimetric radar, Australian Meteorological Magazine, 52, 1, 23-31, 2003.

Lillesand, T. M. and Kiefer, R. W.: Remote sensing and image interpretation, 3rd Edition, John Wiley \& Sons. Inc., 1994.

Lim, S., Chandrasekar, V., and Bringi, V. N.: Hydrometeor classification system using dual-polarization radar measurements: model improvements and in situ verification, 43, 4, 792-801, 2005.

Mendel, J. M.: Fuzzy logic systems for engineering: a tutorial, Proceedings of the IEEE, 83, 345-377, 1995.

Mishchenko, M. I. and Travis, L. D.: Capabilities and limitations of a current Fortran implementation of the T-Matrix method for randomly oriented, rotationally symmetric scatterers, Journal of Quantitative Spectroscopy and Radiative Transfer, 60, 3, 309324, 1998.

Straka, J. M., Zrnić, D. S., and Ryzhkov, A. V.: Bulk hydrometeor classification and quantification using polarimetric radar data: synthesis of relations, J. Appl. Meteorol., 39, 8, 13411372, 2000.

Vivekanandan, J., Zrnić, D. S., Ellis, S. M., Oye, R., Ryzhkov, A. V., and Straka, J.: Cloud microphysics retrieval using S-band dualpolarization radar measurements, Bull. Amer. Meteorol. Soc., 80, 3, 381-388, 1999.

Vulpiani, G., Marzano, F. S., Chandrasekar, V., and Lim, S.: Constrained Iterative Technique with Embedded Neural-Network for Dual-Polarization Radar Correction of Rain Path Attenuation, IEEE Trans. Geosci. Rem. Sensing, 43, 2305-2314, 2005.

Zrnić, D. S., Ryzhkov, A. V., Straka, J., Liu, Y., and Vivekanandan, J.: Testing a procedure for automatic classification of hydrometeor types, J. Atmos. Oceanic Technol., 18, 6, 892-913, 2001. 